\title{
Treatment of Steroid Induced Lipoatrophy with Structural Fat Grafting
}

\author{
Russell B. Stokes \\ Department of Plastic Surgery, Pinehurst Surgical Clinic, Pinehurst, USA. \\ Email: rstokes@pinehurstsurgical.com
}

Received November $29^{\text {th }}, 2011$; revised January $14^{\text {th }}, 2012$; accepted January $25^{\text {th }}, 2012$

\begin{abstract}
Background: Systemic corticosteroid injections are used to treat a variety of medical conditions. Inaccurate placement of intramuscular injections of corticosteroid can result in local lipoatrophy, and a resultant cosmetic deformity. Objective: Describe the condition, and a simple, reproducible method of correcting the volume deficiency and skin changes associated with steroid induced lipoatrophy. Methods: A retrospective chart review was undertaken of all patients treated by the author for steroid induced lipoatrophy between June of 2005 and March of 2011. Results: Structural fat grafting was used to treat 11 patients with steroid induced lipoatrophy during this time period. 10 patients were female and one was male. 9 of the patients had atrophy of the buttock or hip, and 2 had atrophy of the upper extremity. All patients had satisfactory correction of their deformities, and only one of the patients required a second grafting session to achieve an adequate volume for complete correction. Conclusions: Structural fat grafting can eliminate the volume deficiency and skin changes associated with steroid induced lipoatrophy. It is a straightforward, well tolerated method for correcting a problem which can be quite cosmetically deforming.
\end{abstract}

Keywords: Lpoatrophy; Fat Grafting; Steroid

\section{Introduction}

Intramuscular injections of corticosteroids are used to treat a variety of inflammatory medical conditions. These medications are effective at limiting the severity of asthma flares, allergic reactions, and many other conditions encountered by primary care practitioners and specialists on a daily basis. When corticosteroids are injected too superficially, undesired effects, such as lipoatrophy can result. This lipoatrophy can manifest as a small, nearly imperceptible indentation, or it can be quite pronounced, leading to a defect several centimeters in diameter and depth. Patients are often told by the practitioner who administered the steroid that there is nothing which can be done to fix the indentation. This is certainly not the case, and herein a series of 11 patients are reported who underwent structural fat grafting using the Coleman method [1] to repair not only the volume deficiency, but the steroid induced skin changes which often accompany these types of defects.

\section{Methods}

Between June 2005 and March 2011, 11 patients were treated for steroid induced lipoatrophy. These patients ranged in age from 21 - 58 years old, and 10 of the 11 were female. 9 of the defects occurred on the buttocks or hip, and 2 resulted from attempts to place steroid in the deltoid muscle, resulting in lateral arm defects. 10 of the procedures were performed under local anesthesia with or without intravenous sedation, and one was performed with a concomitant augmentation/mastopexy under general anesthesia. Donor sites for fat included the outer thigh, the posterior flank, and the periumbilical abdomen.

After carefully marking the defect in the standing position, the donor sites were outlined as for a typical liposuction procedure. The patients were then placed on the table and positioned to allow access to both the donor site and the defect. The donor site was then injected with $0.5 \%$ lidocaine with 1:200,000 epinephrine. After adequate time for blanching of the skin had been allowed, a $3 \mathrm{~mm}$ incision was made with an 11 blade. A 10cc syringe was used to harvest the fat, along with a $3 \mathrm{~mm}$ Coleman harvest cannula. Gentle retraction of the plunger, as described by Coleman [2] allowed the necessary amount of fat to be harvested. The fat was then centrifuged at 3000 rpm for 3 minutes, allowing the syringe contents to be separated into 3 distinct layers. The superficial oily layer was then poured off, and the deeper saline/blood layer was decanted. The remaining fat was then transferred to 1 cc syringes for injection into the defect. 
The defect was injected with $0.5 \%$ lidocaine with 1:200,000 epinephrine during the centrifugation process, to allow time for vasoconstriction prior to placement of fat grafts. Once the fat was ready for transfer, it was placed into the defect using usually 2 entry points at 90 degrees from one another around the periphery of the defect. The grafts were placed using a blunt 17 gauge Coleman transfer cannula with a single lumen in most cases. For larger defects, a 14 gauge needle was used for a portion of the grafting. Multiple passes are made injecting small aliquots upon withdrawal of the cannula. The volume of fat grafted ranged from 18 cc to 57 cc. Modest overcorrection was targeted to compensate for some fat resorption, which typically occurs. At the completion of the case, the access incisions were closed with 6 - 0 nylon, and the patient was placed into a mildly compressive garment to cover the donor site. An attempt was always made to use a donor site which would allow a single garment to compress the donor site and the recipient site. No perioperative antibiotics were used, and most patients did not require any narcotic analgesics.

\section{Results}

10 of the 11 patients treated were able to be corrected with a single grafting session. One patient, who had a particularly deep defect (Figure 1) required a second session to achieve complete correction, after slightly more than anticipated fat resorption. There were no infections, no hematomas, no problem scars, and to date there have been no instances of fat necrosis or other masses in the recipient sites. No fat migration was noted in any of the cases. Follow up ranged from 7 month to 36 months, with 7 of the patients being followed for over 1 year. All 7 of these patients have maintained their correction (Table 1).

\section{Discussion}

The mechanism by which corticosteroids cause lipoatrophy is not completely understood. A recent study suggests that corticosteroids activate macrophages which then "engulf segments of altered adipose and stromal tissue" [3]. In addition to the lipoatrophy which occurs with injudicious placement of corticosteroid, skin changes can often be seen as well [4]. Thinning of the overlying skin, as well as lightening of the skin can often accompany the volume changes which occur with lipoatrophy. The mechanism for these skin changes involves alteration in the synthesis of both type I and type III collagen, as well as a reduction in the active and latent forms of collagenase [4]. Additionally, melanocyte function appears to be reduced in response to steroid injections in the skin, leading to the hypopigmentation often seen in association with steroid injections. Interestingly, melanocytes are present in adequate numbers at the dermal-epidermal junction in these

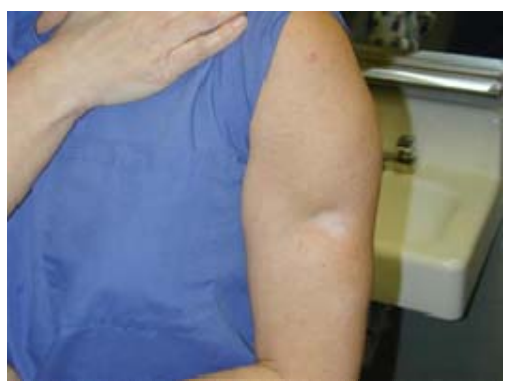

(a)

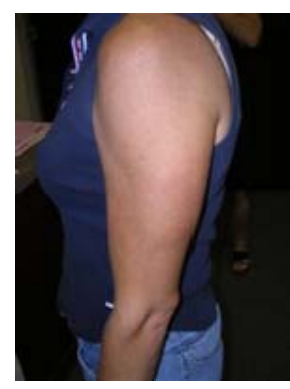

(b)
Figure 1. (a) Appearance of steroid induced atrophy of the arm after triamcinolone injection 14 months prior; (b) 36 months after two fat grafting sessions, separated by 5 months. A total of $18 \mathrm{cc}$ was placed in the first session, and $12 \mathrm{cc}$ in the second session. Note improved skin appearance

Table 1. Complications among 11 patients.

\begin{tabular}{ll}
\hline Hematoma & 0 \\
\hline Numbness & 0 \\
Infection & 0 \\
Palpable fat necrosis & 0 \\
Recurrence of defect & 0 \\
Additional grafting session required & 1 \\
Fat Overgrowth & 0 \\
\hline
\end{tabular}

types of lesions, but their function appears to be impaired, leading to diminished pigment production [5].

Local injection of saline solution has been proposed as a treatment for steroid induced cutaneous atrophy [6], as well as for lipoatrophy associated with saline breast implants which have been injected with steroid solution [7]. The theory proposed is that the saline would re-dissolve the steroid crystals present in the tissues of patients suffering this type of atrophy. To my knowledge, there is no data available on the length of time which steroid might linger in tissues, but I would postulate that the medication is long since gone in the patients described in this study, as most of them presented years after the injection of steroid into the area.

One potential complication of using this technique is the overgrowth of the grafted fat [8,9]. Latoni, et al., reported using fat grafting to treat an area of steroid induced lipoatrophy whereby the patient subsequently had enlargement of the grafted fat many months after the grafting procedure [8]. While this phenomenon has been described previously [9] in fat grafting cases not done for steroid atrophy, I have not observed it using the structural fat grafting technique employed herein.

Interestingly, the cutaneous atrophy often seen with these lipoatrophy defects improves dramatically with fat grafting, as evidenced in the post-procedure photographs 
shown here. The same phenomenon has been described by other authors $[2,8]$, and grafted fat has been shown to influence the tissue into which it is placed when done so to treat conditions such as radiation dermatitis [10]. Whether adipose-derived stem cells are responsible for this behavior, or some other effect of the grafted fat, such as increased vasculogenesis [11], remains to be determined.

\section{Conclusions}

A straightforward method of correcting steroid induced lipoatrophy is presented. The results have been very good when following a standardized method of fat harvest and injection, structural fat grafting.

\section{REFERENCES}

[1] S. R. Coleman, "Structural Fat Grafts: The Ideal Filler?” Clinics in Plastic Surgery, Vol. 28, No. 110, 2002, pp. 1731-1745.

[2] S. R. Coleman, "Structural Fat Grafting: More than an Ideal Filler?” Plastic \& Reconstructive Surgery, Vol. 118, No. 3S, 2006, pp. 108s-120s. doi:10.1097/01.prs.0000234610.81672.e7

[3] I. Ahmed, "Post-Injection Involutional Lipoatrophy: Ultrastructural Evidence for an Activated Macrophage Phenotype and Macrophage Related Involution of Adipocytes," American Journal of Dermatopathology, Vol. 28 No. 4, 2006, pp. 334-337. doi:10.1097/00000372-200608000-00008

[4] Y. Oishi, Z. W. Fu, Y. Ohnuki, et al., "Molecular Basis of the Alteration in Skin Collagen Metabolism in Response to in Vivo Dexamethasone Treatment: Effects on the Synthesis of Collagen Type I and III, Collagenase, and
Tissue Inhibitors of Metalloproteinases,” British Journal of Dermatology, Vol. 147, No. 5, 2002, pp. 859-868. doi:10.1046/j.1365-2133.2002.04949.x

[5] P. Venkatesan and W. L. Fangman, "Linear Hypopigmentation and Cutaneous Atrophy Following Intra-Articular Steroid Injections for de Quervain's Tendonitis," Journal of Drugs in Dermatology, Vol. 8, 2009, pp. 492493.

[6] P. R. Shumaker, J. Rao and M. P. Goldman, “Treatment of Local, Persistent Cutaneous Atrophy Following Corticosteroid Injection with Normal Saline Infiltration,” Dermatologic Surgery, Vol. 10, 2005, pp. 1340-1343.

[7] M. A. Shiffman, "New Treatment of Steroid-Induced Fat Atrophy,” Plastic \& Reconstructive Surgery, Vol. 109, No. 7, 2002, pp. 2609-2610. doi:10.1097/00006534-200206000-00090

[8] J. D. Latoni, D. M. Marshall and S. A. Wolfe, "Overgrowth of Fat Autotransplanted for Correction of Localized Steroid-Induced Atrophy,” Plastic \& Reconstructive Surgery, Vol. 106, No. 7, 2000, pp. 1566-1569. doi:10.1097/00006534-200012000-00018

[9] R. Ellenbogen, "Free Autogenous Pearl Fat Grafts in the Face: A Preliminary Report of a Rediscovered Technique,” Annals of Plastic Surgery, Vol. 16, No. 3, 1986, p. 179. doi:10.1097/00000637-198603000-00001

[10] G. Rigotti, A. Marchi, M. Galie, et al., "Clinical Treatment of Radiotherapy Tissue Damages y Lipoaspirates Transplant: A Healing Process Mediated by Adipose Derived Stem Cells,” Plastic \& Reconstructive Surgery, Vol. 119, No. 5, 2007, pp. 1409-1422. doi:10.1097/01.prs.0000256047.47909.71

[11] J. G. Neels, T. Thinnes and D. J. Loskutoff, “Angiogenesis in an in Vivo Model of Adipose Tissue Development," Journal of the Federation of American Societies for Experimental Biology, Vol. 18, No. 9, 2004, pp. 983-985. 